\title{
Aspectos éticos e legais da assistência religiosa em hospitais psiquiátricos
}

\author{
Ethical and legal aspects of religious assistance in psychiatric hospitals \\ Camilla Casaletti Braghetta1 ${ }^{1}$ Giancarlo Lucchetti², Frederico Camelo Leão ${ }^{3}$, CÂndido Vallada ${ }^{4}$, Homero Vallada ${ }^{5}$, \\ QUIRINO CORDEIRO 6
}

\author{
1 Programa de Integração Saúde Mental e Espiritualidade (PRISME) do Hospital João Evangelista (HOJE). \\ 2 HOJE, Departamento de Pesquisa da Associação Médico-Espírita de São Paulo. \\ ${ }_{3}$ HOJE, Programa de Saúde Espiritualidade e Religiosidade (ProSER) do Instituto de Psiquiatria do Hospital das Clínicas da Faculdade de Medicina da Universidade de São Paulo (FMUSP). \\ ${ }_{4}$ PRISME do HOJE. \\ 5 HOJE, Departamento de Psiquiatria da FMUSP. \\ ${ }^{6}$ Departamento de Psiquiatria e Psicologia Médica da Faculdade de Ciências Médicas da Santa Casa de São Paulo.
}

Recebido: $16 / 11 / 2010$ - Aceito: 4/4/2011

\begin{abstract}
Resumo
A assistência religiosa a pacientes com transtornos psiquiátricos graves internados é um problema pouco discutido entre psiquiatras. Aspectos legais asseguram o direito à assistência religiosa aos pacientes, no entanto alguns aspectos específicos conduzem a reflexão e cautela em determinadas situações. Assim, objetiva-se com o presente trabalho discutir os aspectos éticos, legais e científicos da assistência religiosa em hospitais psiquiátricos. Serão abordados também alguns comentários sobre os conceitos de autonomia, beneficência e não maleficência, contidos nos princípios bioéticos. Ao final, é apresentada a experiência do Hospital João Evangelista na abordagem de tais casos. Como conclusão, situações de conflito ético e legal podem surgir, pois os pacientes têm direito a receber assistência religiosa, porém podem apresentar manifestações psicopatológicas que poderiam levar a prejuízo de seu quadro clínico diante da abordagem religiosa. A orientação dos familiares e as relações entre hospital, médico, paciente e família são essenciais para a resolução de tais conflitos.
\end{abstract}

Braghetta CC, et al. / Rev Psiq Clín. 2011;38(5):189-93

Palavras-chave: Espiritualidade, religião e medicina, psiquiatria.

\begin{abstract}
The religious assistance to inpatients with severe psychiatric disorders is a problem rarely discussed among psychiatrists. Legal aspects ensure the right to religious assistance to such patients, although some specific aspects lead to reflection and caution in certain situations. Thus, the main of the work is to discuss ethical, legal, scientific and religious aspects of religious assistance in psychiatric hospitals. It will also address some comments on the concepts of autonomy, beneficence and nonmaleficence, contained in bioethical principles. Finally, it will be presented the experience of the João Evangelista Hospital approach on such cases. In conclusion, ethical and legal conflicts can appear because patients have the right to receive a religious attendance. However, in some conditions, this assistance could lead to a worse in patient's clinical status. Family orientation and doctor-patient-hospital relationship are essential to resolve these conflicts.
\end{abstract}

Braghetta CC, et al. / Rev Psiq Clín. 2011;38(5):189-93

Keywords: Spirituality, religion and medicine, psychiatry.

\section{Introdução}

A religiosidade e a espiritualidade são dimensões integrantes da experiência social e cultural humana e, portanto, da vivência cotidiana de grande parte da população. Um estudo da Organização Mundial da Saúde (OMS) investigou 5.087 pessoas em 18 países, e, dentre os países cristãos fora da África, o Brasil teve a maior porcentagem de entrevistados que indicaram ser "moderadamente" ou "extremamente" religiosos (80\% a $90 \%)^{1}$.

Dados do censo demográfico realizado no ano de 2000 mostram que parcela superior a $90 \%$ da população brasileira possui alguma denominação religiosa e grande parte da população brasileira possui alguma forma de expressão espiritual ou envolvimento religioso ${ }^{2}$.

Esses dados são correlatos a um recente levantamento populacional nacional envolvendo 3.007 participantes de uma amostra representativa da população brasileira ${ }^{3}$. De acordo com o estudo, apenas $5 \%$ dos brasileiros declararam não ter religião, $83 \%$ consideraram a religião muito importante para sua vida e $37 \%$ frequentavam algum serviço religioso pelo menos uma vez por semana. As filiações religiosas mais frequentes foram Catolicismo (68\%), Protestante/ Evangélica (23\%) e Espiritismo Kardecista (2,5\%).

A religiosidade de um indivíduo, segundo Allport e Ross ${ }^{4}$, pode ser de orientação intrínseca ou extrínseca. Na religiosidade do tipo intrínseca, as pessoas têm na religião seu bem maior, sendo outras necessidades vistas como de menor importância. Em geral, outras questões são colocadas em harmonia com sua orientação e crença religiosa. Já na religiosidade extrínseca, a religião é um meio utilizado pelo indivíduo para obter outros fins ou interesses, sociabilidade e distração, status e autoabsolvição.

As crenças e serviços religiosos, muitas vezes, podem auxiliar pacientes a conseguirem enfrentar melhor as adversidades provenientes de um processo de adoecimento. Quando indivíduos se voltam para a religião para lidar com o estresse, é caracterizado o chamado "coping" religioso-espirituals,6, que é definido como "o uso de crenças e comportamentos religiosos para facilitar a solução de problemas e prevenir ou aliviar as consequências emocionais negativas de circunstâncias de vida estressantes". A quase totalidade das pesquisas em populações saudáveis sugere que as crenças e práticas religiosas estão associadas com maior bem-estar psicológico, maior "coping" e melhor saúde mental7. Essas associações entre religiosidade e melhor saúde mental são encontradas de modo mais marcante em situações de alto estresse.

Esses achados também são verificados entre pacientes com transtornos psiquiátricos, já que eles enfrentam enorme estresse ambiental e psicossocial em razão de seus transtornos, necessitando de estratégias eficazes de enfrentamento.

Estudos sugerem que a religiosidade e a espiritualidade podem ter efeito protetor sobre a saúde e parecem influenciar a saúde física 
e mental de várias maneiras, por exemplo, por meio de regras de convivência e do desestímulo a comportamentos prejudiciais à saúde, como abuso de álcool e drogas, comportamento violento ou sexual de risco; por meio do uso de crenças religiosas como forma de lidar com situações adversas, como uma doença, e por meio da criação de uma rede de suporte socials,9.

Por outro lado, alguns estudos também indicam associação entre envolvimento religioso e maior psicopatologia ${ }^{10}$. Essa relação positiva e negativa da religiosidade no paciente com transtornos mentais leva a questões éticas importantes durante as abordagens espirituais.

\section{Religiosidade e pacientes com transtornos mentais: relações positivas}

Diversos estudos têm demonstrado relações positivas entre religiosidade e pacientes com transtornos mentais. Koenig et al., em 1998, avaliaram pacientes diagnosticados com depressão maior e mostraram que a religiosidade intrínseca estava relacionada a menor tempo para remissão de depressão ${ }^{11}$, resultado compatível com outro estudo de 2007 em que pacientes com maior envolvimento religioso comunitário tiveram remissões mais rápidas do transtorno depressivo ${ }^{12}$.

De forma semelhante, a maioria dos estudos tem observado relação inversa entre religiosidade e transtorno de ansiedade generalizada (TAG). Em 2004, Shreve-Neiger e Edelstein confirmaram essas observações em uma revisão crítica de 17 artigos, alertando, no entanto, para a importância dos cuidados metodológicos e conceituais na realização dos estudos ${ }^{13}$. Há também relatos de equivalência terapêutica entre intervenções espirituais e cognitivo-comportamentais para o tratamento do $\mathrm{TAG}^{14}$.

Quanto ao uso de drogas, estudos têm demonstrado que os pacientes dependentes de substâncias possuem menores níveis de religiosidade e que o engajamento espiritual parece estar relacionado com melhor recuperação do indivíduo ${ }^{15}$. Alguns grupos, como os Alcoólicos Anônimos, utilizam aspectos espirituais em sua terapêutica como a busca pessoal, a prece, a meditação e o contato com Deus, tendo bons resultados para os frequentadores regulares ${ }^{16}$.

Quanto à prevalência de suicídio, estudos têm demonstrado que a taxa de suicídios entre indivíduos que não frequentam serviços religiosos é de quatro a sete vezes maior do que entre aqueles que frequentam ${ }^{17,18}$.

Alguns estudos em pacientes com esquizofrenia observam correlação entre a falta de envolvimento religioso, maior re-hospitalização ${ }^{19}$ e maior deterioração clínica no seguimento desses indivíduos ${ }^{20}$. Os delírios religiosos não prejudicaram a resposta ao tratamento quando comparados a outros pacientes ${ }^{21}$.

A equipe do Dr. Huguelet da Universidade de Genebra ${ }^{22,23}$ contribuiu com dois recentes estudos nessa interface. O primeiro 22 avaliou as diferenças entre o coping religioso/espiritual (R/E) positivo e negativo na forma de lidar com o quadro de esquizofrenia ou transtorno esquizoafetivo. A importância da espiritualidade foi preditiva de menor presença de sintomas negativos e melhor avaliação clínica global, funcionamento social e qualidade de vida. Cerca de $83 \%$ dos pacientes apresentavam coping $\mathrm{R} / \mathrm{E}$ positivo e apenas $14 \%$ dos pacientes apresentavam coping R/E negativo. O estudo apontou que o uso positivo/benéfico da espiritualidade seria um preditor de melhores resultados. Entretanto, em alguns casos, a espiritualidade e a religiosidade foram fonte de sofrimento dos pacientes.

O segundo estudo ${ }^{23}$ analisou a aceitação de avaliação espiritual por pacientes e médicos, as sugestões para o tratamento que surgiram a partir dessa avaliação, a adesão ao tratamento e a satisfação com o atendimento. Concluiu-se que a avaliação espiritual pode suscitar importantes questões clínicas no tratamento de pacientes com esquizofrenia crônica. Segundo os autores, fatores culturais como religião e espiritualidade devem ser considerados no início de treinamento clínico.

Ainda em relação à esquizofrenia e à religião, uma revisão de literatura ${ }^{24}$ aponta que alguns pacientes são ajudados por sua comunidade de fé, apoiados por atividades espirituais, confortados e fortalecidos por suas crenças. Da mesma forma, outros pacientes são rejeitados por sua comunidade religiosa, sobrecarregados pelas atividades espirituais e apresentam-se decepcionados em relação a suas crenças. No entanto, levar em consideração a religiosidade parece ser relevante para o tratamento de pessoas com esquizofrenia, na medida em que pode ajudar a reduzir sintomas psicopatológicos, para ajudar a lidar com as dificuldades e promover a recuperação.

Evidências têm mostrado que as pessoas tornam-se ainda mais religiosas quando estão doentes, tanto física como mentalmente ${ }^{25,26}$. Em situações de alto estresse psicológico, a religião é frequentemente utilizada para auxiliar a lidar com situações de sofrimento ou a se adaptar a elas. Assim, não é surpreendente que pacientes psicóticos e portadores de transtornos mentais graves e persistentes (i.e., condições altamente estressantes) possam também ser bastante religiosos ${ }^{10}$.

\section{Religiosidade e pacientes com transtornos mentais: relações negativas}

Apesar de a maioria dos estudos apontar para associações positivas, a religiosidade pode também ter relações negativas nos pacientes com transtornos psiquiátricos. A religiosidade negativa, como a luta religiosa (religious struggle) que envolve sentimento de abandono e punição por Deus, está correlacionada à maior mortalidade ${ }^{27}$.

Para alguns indivíduos, o diagnóstico de doença grave ou outros eventos adversos da vida podem precipitar um período de conflito religioso. Um estudo incluiu pacientes diabéticos, portadores de insuficiência cardíaca congestiva e oncológicos. Cerca de $15 \%$ dos participantes relataram níveis moderados a altos de conflitos religiosos. A luta religiosa foi associada com maiores níveis de sintomas depressivos e problemas emocionais em todos os três grupos de pacientes ${ }^{28}$. Alguns estudos também demonstram uma relação entre maior frequência a cultos e sintomas ansiosos ${ }^{29}$.

Quanto a pacientes com esquizofrenia, Getz et al. avaliaram 131 indivíduos nos Estados Unidos e concluíram que a afiliação religiosa (neste caso, protestante ou católica) foi independente da gravidade do delírio religioso quando comparada aos que não possuíam afiliação religiosa $)^{30}$.

Em contraponto, Nucci e Dalgalarrondo ${ }^{31}$ relataram ocorrências de enucleações oculares em seis pacientes psiquiátricos ao longo do período de 10 anos em um hospital universitário brasileiro. Delírios religiosos foram um fator significativo em muitos desses casos, em que o paciente agia conforme indica o livro de Mateus (5:29): "Se o teu olho direito te escandalizar, arranca-o e atira-o para longe de ti, pois te é melhor que se perca um dos teus membros do que seja todo o teu corpo lançado no inferno". Esses pacientes foram diagnosticados como uma exacerbação aguda de esquizofrenia.

Estima-se que pacientes psicóticos possuem prevalência de delírios religiosos variando entre $7 \%$ e $43 \%{ }^{10}$.

\section{Intervenções espirituais em pacientes com transtornos mentais}

Já que muitos pacientes com transtornos mentais graves usam a religião para lidar com seus problemas, acredita-se que intervenções religiosas ou espirituais possam ser úteis nesse contexto.

Peres et al. ${ }^{32}$ discutem, em artigo de revisão, o impacto da subjetividade, dos estados de consciência e das percepções influenciadas pela religiosidade/espiritualidade na saúde mental e a importância de a psicoterapia voltar-se a clientes e seus respectivos sistemas de crenças, desenvolvendo modelos que mobilizem esperança e potencializem capacidades de superação.

Fallot descreve como as necessidades espirituais dos pacientes com transtornos mentais graves podem ser abordadas como parte do tratamento ${ }^{33}$. Dentro dessa abordagem complementar de reabilitação, incluem-se a obtenção da história espiritual do paciente, a existência de grupos de espiritualidade, a psicoterapia com abordagem da espiritualidade e a conexão entre comunidades religiosas e recursos espirituais. 
Alguns estudos têm demonstrado que abordagens religiosas e espirituais levariam a bons resultados no tratamento de pacientes com transtornos mentais. Philips et al. ${ }^{34}$ descrevem um programa para as pessoas com transtornos mentais graves que objetivou lidar com questões espirituais/religiosas por meio de atendimento de grupo semiestruturado de intervenção psicoeducacional, em que os participantes discutiam sobre recursos religiosos, conflitos espirituais, perdão e esperança.

Kehoe $^{35}$ descreve a experiência adquirida ao longo de 18 anos de condução de um grupo de terapia para pacientes com transtornos mentais crônicos que incide sobre as crenças e valores espirituais. Segundo o autor, autoconsciência, tolerância da diversidade e respeito pelas crenças dos outros foram observados nos participantes com $o$ atendimento.

Leão realizou estudo em instituição para pacientes portadores de deficiência mental, comparando grupo experimental submetido à prática espiritual com grupo controle. A análise dos resultados confirmou a hipótese de que o uso de práticas espirituais apresenta resultados positivos na evolução clínica e comportamental dessa população ${ }^{36}$.

Entretanto, há a preocupação de que essas intervenções possam interferir na recuperação de pessoas com transtornos mentais graves de forma negativa, podendo complicá-la, especialmente se estão presentes alucinações e/ou delírios religiosos ${ }^{10}$. Salib e Youakim ${ }^{37}$ realizaram estudo cujo objetivo foi investigar se havia associação entre o recebimento da cura espiritual e a ocorrência de reagudizações de sintomas psicóticos em esquizofrenia em uma amostra de pacientes idosos de etnia egípcia. $\mathrm{O}$ método utilizado foi um estudo caso-controle comparando pacientes com o diagnóstico de esquizofrenia que receberam a cura espiritual e sem cura espiritual, em termos da ocorrência de recaídas durante determinado período. Os pacientes que receberam a cura espiritual recaíram com mais frequência do que aqueles que não o receberam.

Assim, a distinção entre curas espirituais e intervenções espirituais deve ser feita até que mais estudos confirmem esses achados. Entretanto, até o momento, nenhuma intervenção espiritual, tanto individual como em grupo, teve ainda sua eficácia e segurança testadas em pacientes com esquizofrenia, de forma objetiva e rigorosa em ensaios clínicos randomizados.

\section{Aspectos éticos e legais}

No Brasil, a Constituição Federal (CF), em seu artigo $5^{\circ}$, a Lei Federal $n^{\circ}$ 9.982/2000 e leis estaduais asseguram e regulamentam a assistência religiosa em hospitais, desde que realizada em comum acordo com pacientes e seus familiares, no caso de pacientes que não estejam no gozo de suas faculdades mentais. A Constituição prevê que "é assegurada, nos termos da lei, a prestação de assistência religiosa nas entidades civis e militares de internação coletiva" (CF, art. 5, VII). Em 2000, a Lei Federal n 9.982/2000 ("Assistência Religiosa nas Entidades Hospitalares Públicas e Privadas, e nos Estabelecimentos Prisionais Civis e Militares") veio regulamentar essa prática, dispondo sobre a prestação de assistência religiosa nas entidades hospitalares públicas e privadas, bem como nos estabelecimentos prisionais civis e militares. Em seu artigo $1^{\circ}$, a lei declara que "aos religiosos de todas as confissões assegura-se o acesso aos hospitais da rede pública ou privada, bem como aos estabelecimentos prisionais civis ou militares, para dar atendimento religioso aos internados, desde que em comum acordo com estes, ou com seus familiares no caso de doentes que já não mais estejam no gozo de suas faculdades mentais". A realização da assistência religiosa, portanto, não passa pela decisão da equipe terapêutica do Hospital onde o paciente se encontra internado. Vale ressaltar que o artigo $1^{\circ}$ da lei possuía um parágrafo único, porém que fora vetado. $\mathrm{O}$ texto de tal parágrafo dizia: "a prestação de assistência religiosa não será permitida se, a juízo das entidades suprarreferidas, houver risco à vida ou à saúde do interno ou do religioso". Desse modo, no que tange à assistência religiosa aos pacientes internados devido a transtornos mentais graves, por lei, basta que haja a concordância dos próprios pacientes, ou de seus familiares, "no caso de doentes que não estejam no gozo de suas faculdades mentais", para que tal prática possa ser realizada.
Entretanto, como descrito anteriormente, há preocupação que as intervenções religiosas possam prejudicar o tratamento de pacientes com transtornos mentais graves, especialmente quando da vigência de sintomas psicóticos como alucinações ou delírios religiosos.

Conforme apresentado neste artigo, diversos estudos têm mostrado resultados conflitantes quanto ao benefício da assistência religiosa a pacientes com transtornos psicóticos. Gearing et al. sugerem que religião pode agir como fator de risco ou de proteção à medida que pode interagir com sintomas da esquizofrenia como delírios e alucinações. Influências culturais tendem a confundir a associação de religião e da esquizofrenia. No entanto, os autores apontam que observar a relação entre religião e esquizofrenia pode ser benéfico tanto para os profissionais de saúde quanto aos pesquisadores com a melhora da adesão ao tratamento, a valorização dos aspectos de proteção e para minimizar os riscos associados ${ }^{38}$.

Em estudo recente realizado em pacientes chineses, foi observado que os pacientes com afiliação religiosa apresentaram menor preferência para tratamento psiquiátrico. Os indivíduos com delírios religiosos/alucinações eram mais propensos a aceitar a cura mágico-religiosa e não eram satisfeitos com o tratamento psiquiátrico ${ }^{39}$.

Diante disso, situações de conflito ético podem surgir, pois os pacientes têm direito a receber assistência religiosa, porém podem apresentar manifestações psicopatológicas que poderiam levar a prejuízo de seu quadro clínico diante da abordagem religiosa.

Considera-se a autonomia como a necessidade de respeitar o direito do indivíduo à autodeterminação e às decisões que toma em relação aos cuidados de saúde e de apoio social, desde que esse indivíduo possua a capacidade para fazê-lo. Outro princípio bioético, a beneficência, consiste na necessidade de considerar os benefícios potenciais que podem resultar de uma intervenção em comparação aos riscos potenciais. A não maleficência é a necessidade de não causar dano ao indivíduo ${ }^{40}$.

Muitos pacientes consideram importante sua dimensão espiritual e gostariam de receber apoio nesse sentido quando internados. É papel do profissional de saúde facilitar essa assistência, respeitando os princípios da autonomia e beneficência.

No caso de presença de sintomas psicóticos, em que há possibilidade de agravamento do quadro com essa intervenção espiritual/ religiosa, pode-se sugerir ao paciente e familiares que não seja feita a assistência, seguindo o princípio bioético da não maleficência. No entanto, assim que o quadro psiquiátrico estabilizar, é importante que a autonomia do paciente seja retomada e estimulada.

O objetivo das intervenções contemporâneas em saúde mental é preservar e estimular a autonomia das pessoas portadoras de transtornos mentais. No entanto, é imprescindível que essas práticas estejam igualmente congruentes com os princípios bioéticos de beneficência e não maleficência. É necessário levar em consideração a condição de vulnerabilidade em que se encontra esse paciente, pois, além de esse ser portador de um transtorno mental, com a possibilidade de ter agravamento de seu quadro, também está em situação de internação, submetido às normas de uma instituição de saúde. Sendo assim, é necessário construir novas propostas de cuidado em saúde mental, sempre de forma congruente aos princípios bioéticos.

Assim sendo, a questão fundamental a ser respondida é saber quando um paciente atua de maneira autônoma ou se, por qualquer questão, está com essa competência reduzida. Desse modo, precisa-se sempre tentar estabelecer qual o limite entre o respeito à decisão autônoma de um paciente e a proteção àqueles que estão com sua autonomia, mesmo que momentaneamente, comprometida ${ }^{41}$. Por exemplo, indivíduos portadores de transtornos mentais graves, em determinados momentos do curso de seus quadros clínicos, podem apresentar sua autonomia reduzida, o que pode influenciar em seus processos de tomada de decisão. Pacientes que apresentam determinados transtornos mentais podem apresentar comprometimento em sua competência para decidir de maneira soberana ${ }^{42,43}$.

Eike-Henner relata ser um equívoco o fato de se tratar da mesma maneira um indivíduo autônomo e outro com sua autonomia comprometida $^{42}$. Com isso, segundo o autor, corre-se o risco de punir e prejudicar o paciente por conta de sua incompetência em 
tomar decisões autônomas. Gessert coloca, ainda, que a extremada importância que se vem dando nos últimos tempos à autonomia do paciente pode resultar em situação desfavorável para ele ${ }^{44}$. O direito à autonomia deve ser cada vez mais respeitado, porém em situações nas quais se apresentam pacientes adultos lúcidos e bem informados. Entretanto, muitas vezes pacientes extremamente vulnerados são colocados em situações nas quais não conseguem agir de maneira autônoma. A simples aplicação do princípio bioético da autonomia a pacientes portadores de transtornos mentais graves com competência comprometida para tomar decisões autônomas pode ser considerada uma distorção ${ }^{41}$. Esse contexto pode exigir postura clínica baseada nos princípios bioéticos da beneficência e não maleficência por parte da equipe terapêutica, seguindo uma ótica de cuidados ao paciente. As ideias que estão na base dos princípios bioéticos da beneficência e não maleficência são a benevolência, a não malevolência e o altruís$\mathrm{mo}^{41}$. Atitudes que têm por base tais princípios buscam garantir que os interesses dos pacientes com autonomia comprometida sejam alcançados. Tais princípios constituíam-se na base da ética hipocrática.

Entretanto, vale ressaltar que os princípios bioéticos não devem ser considerados de maneira hierárquica, devendo ser encarados prima facie, a saber, a opção pela utilização de um deles em detrimento do outro deve acontecer somente depois de reflexão sobre o caso concreto que se avalia ${ }^{41}$.

\section{Modelo do Hospital João Evangelista - São Paulo}

Como visto, integrar um suporte espiritual para pacientes com transtornos mentais graves é um desafio que engloba questões médicas, éticas e legais. O Programa de Integração Saúde Mental e Espiritualidade (PRISME) foi criado pelo Hospital João Evangelista de São Paulo, Brasil, para cuidar da assistência religiosa de seus pacientes internados com transtornos mentais graves. Como preceitos básicos, o programa oferece: (a) suporte para os pacientes e seus familiares receberem assistência religiosa no momento da internação; (b) se solicitado, o programa faz a intermediação com o ministro da religião do paciente e da família; (c) caso o paciente e seus familiares optem pela assistência, o programa respeita a decisão e trabalha na orientação deles, bem como dos ministros religiosos, respeitando a autonomia deles.

No entanto, em situações nas quais os pacientes apresentam sintomas psicóticos, especialmente com conteúdos religiosos, eles e seus familiares são desaconselhados quanto à realização de assistência religiosa (princípio da não maleficência), postergando-a para um momento futuro, quando tais sintomas estiverem remitidos.

Entretanto, obedecendo ao princípio bioético da autonomia e às determinações legais, caso os pacientes com sintomas psicóticos, em conjunto com seus familiares, mesmo assim optarem pela assistência religiosa no contexto descrito acima, o hospital acata a decisão e auxilia-os no seu recebimento, respeitando sua autonomia de decisão, porém tentando intervir com o objetivo de evitar prejuízos aos pacientes.

Intervenções espirituais, especialmente quando aplicadas em grupo, podem influenciar o curso dos transtornos mentais graves de vários modos, incluindo fornecimento de apoio, focalizando as suas preocupações espirituais e aumentando as suas habilidades para relacionar-se com outros.

Infelizmente, há muito sobre a relação entre religião e transtornos psicóticos que permanece desconhecido, apontando para a necessidade de mais pesquisas. Porém, o conhecimento vigente já justifica ao menos alguns passos cautelosos adiante. Colher uma cuidadosa história espiritual, apoiar o envolvimento religioso não psicótico e considerar intervenções de grupos espiritualmente fundamentadas para pacientes que tenham essa inclinação parecem ser interessantes caminhos a ser trilhados ${ }^{10}$.

\section{Conclusão}

Estudos têm sugerido que crenças religiosas são usadas por pacientes com transtornos mentais graves para lidar com o estresse que seus quadros clínicos causam. Assim, intervenções religiosas podem ser úteis no auxílio a tais pacientes. Entretanto, há preocupação que essas intervenções possam prejudicar o tratamento de pacientes com transtornos mentais graves, especialmente na vigência de alucinações ou delírios religiosos.

Diversos estudos têm mostrado resultados conflitantes quanto ao benefício da assistência religiosa a pacientes com transtornos psicóticos. Diante disso, situações de conflito ético e legal podem surgir, pois os pacientes têm direito a receber assistência religiosa, porém podem apresentar manifestações psicopatológicas que poderiam levar a prejuízo de seu quadro clínico diante da abordagem religiosa. A orientação dos familiares e as relações entre hospital, médico, paciente e família são essenciais para a resolução de tais conflitos.

\section{Referências}

1. A cross-cultural study of spirituality, religion, and personal beliefs as components of quality of life. Soc Sci Med. 2006;62:1486-97.

2. Almeida RD. Religião na metrópole paulista. Rev Bras Ciênc Soc 2004;19:15-27.

3. Moreira-Almeida A, Pinsky I, Zaleski M, Laranjeira R. Envolvimento religioso e fatores sociodemográficos: resultados de um levantamento nacional no Brasil. Rev Psiq Clín. 2010;37:12-5.

4. Allport GW, Ross JM. Personal religious orientation and prejudice. J Pers Soc Psychol. 1967;5:432-43.

5. Pargament K. The psychology of religion and coping: theory, research, practice: The Guilford Press; 2001

6. Peres MF, Lucchetti G. Coping strategies in chronic pain. Curr Pain Headache Rep. 2010;14:331-8.

7. Moreira-Almeida A, Neto FL, Koenig HG. Religiousness and mental health: a review. Rev Bras Psiquiatr. 2006;28:242-50.

8. Stroppa A, Moreira-Almeida A. Religiosidade e espiritualidade no transtorno bipolar do humor. Rev Psiq Clín. 2009;36:190-6.

9. Koenig HG, McCullough ME, Larson DB. Handbook of religion and health: Oxford University Press, USA; 2001.

10. Koenig HG. Religião, espiritualidade e transtornos psicóticos. Rev Psiq Clín. 2007;34:95-104.

11. Koenig HG, George LK, Peterson BL. Religiosity and remission of depression in medically ill older patients. Am J Psychiatry. 1998;155:536-42.

12. Koenig HG. Religion and remission of depression in medical inpatients with heart failure/pulmonary disease. J Nerv Ment Dis. 2007;195:389-95.

13. Shreve-Neiger AK, Edelstein BA. Religion and anxiety: a critical review of the literature. Clin Psychol Rev. 2004;24:379-97.

14. Koszycki D, Raab K, Aldosary F, Bradwejn J. A multifaith spiritually based intervention for generalized anxiety disorder: a pilot randomized trial. J Clin Psychol. 2010;66:430-41.

15. Miller WR. Researching the spiritual dimensions of alcohol and other drug problems. Addiction. 1998;93:979-90.

16. Matching alcoholism treatments to client heterogeneity: project MATCH three-year drinking outcomes. Alcohol Clin Exp Res. 1998;22:1300-11.

17. Nisbet PA, Duberstein PR, Conwell Y, Seidlitz L. The effect of participation in religious activities on suicide versus natural death in adults 50 and older. J Nerv Ment Dis. 2000;188:543-6.

18. Hilton SC, Fellingham GW, Lyon JL. Suicide rates and religious commitment in young adult males in Utah. Am J Epidemiol. 2002;155:413-9.

19. Chu CC, Klein HE. Psychosocial and environmental variables in outcome of black schizophrenics. J Natl Med Assoc. 1985;77:793-6.

20. Verghese A, John JK, Rajkumar S, Richard J, Sethi BB, Trivedi JK. Factors associated with the course and outcome of schizophrenia in India: results of a two-year multicentre follow-up study. Br J Psychiatry. 1989;154:499-503.

21. Siddle R, Haddock G, Tarrier N, Faragher E. Religious beliefs and religious delusions: response to treatment in schizophrenia. Mental Health Religion Culture. 2004;7:211-23.

22. Mohr S, Perroud N, Gillieron C, Brandt PY, Rieben I, Borras L, et al. Spirituality and religiousness as predictive factors of outcome in schizophrenia and schizo-affective disorders. Psychiatry Res. 2011;186(2-3):177-82. Epub 2010 Sep 24.

23. Huguelet P, Mohr S, Betrisey C, Borras L, Gillieron C, Marie AM, et al A randomized trial of spiritual assessment of outpatients with schizophrenia: patients' and clinicians' experience. Psychiatr Serv. 2011;62:79-86.

24. Mohr S, Huguelet P. The relationship between schizophrenia and religion and its implications for care. Swiss Med Wkly. 2004;134:369-76. 
25. Ironson G, Stuetzle R, Fletcher MA. An increase in religiousness/spirituality occurs after HIV diagnosis and predicts slower disease progression over 4 years in people with HIV. J Gen Intern Med. 2006;21(Suppl 5):S62-8.

26. Brown SL, Nesse RM, House JS, Utz RL. Religion and emotional compensation: results from a prospective study of widowhood. Pers Soc Psychol Bull. 2004;30:1165-74.

27. Pargament KI, Koenig HG, Tarakeshwar N, Hahn J. Religious struggle as a predictor of mortality among medically ill elderly patients: a 2 -year longitudinal study. Arch Intern Med. 2001;161:1881-5.

28. Fitchett G, Murphy PE, Kim J, Gibbons JL, Cameron JR, Davis JA. Religious struggle: prevalence, correlates and mental health risks in diabetic, congestive heart failure, and oncology patients. Int J Psychiatry Med. 2004;34:179-96.

29. Baker M, Gorsuch R. Trait anxiety and intrinsic-extrinsic religiousness. J Sci Stud Relig. 1982;21:119-22.

30. Getz GE, Fleck DE, Strakowski SM. Frequency and severity of religious delusions in Christian patients with psychosis. Psychiatry Res. 2001;103:87-91.

31. Nucci MG, Dalgalarrondo P. Self-mutilation: report of six cases of enucleation. Rev Bras Psiquiatr. 2000;22:80-6.

32. Peres JFP, Simão MJP, Nasello AG. Espiritualidade, religiosidade e psicoterapia. Rev Psiq Clín. 2007;34:136-45.

33. Fallot R. Spirituality and religion in psychiatric rehabilitation and recovery from mental illness. Int Rev Psychiatry. 2001;13:110-6.

34. Phillips 3rd RE, Lakin R, Pargament KI. Development and implementation of a spiritual issues psychoeducational group for those with serious mental illness. Community Ment Health J. 2002;38:487-95.
35. Kehoe NC. A therapy group on spiritual issues for patients with chronic mental illness. Psychiatr Serv. 1999;50:1081-3.

36. Leão FC, Lotufo Neto F. Spiritual practices in an institution for mental disabilities. Rev Psiq Clín. 2007;34:54-9.

37. Salib E, Youakim S. Spiritual healing in elderly psychiatric patients: a case-control study in an Egyptian psychiatric hospital. Aging Ment Health. 2001;5:366-70.

38. Gearing RE, Alonzo D, Smolak A, McHugh K, Harmon S, Baldwin S. Association of religion with delusions and hallucinations in the context of schizophrenia: implications for engagement and adherence. Schizophr Res. 2011;126(1-3):150-63. Epub 2010 Dec 4.

39. Huang CL, Shang CY, Shieh MS, Lin HN, Su JC. The interactions between religion, religiosity, religious delusion/hallucination, and treatment-seeking behavior among schizophrenic patients in Taiwan. Psychiatry Res. 2011;187(3):347-53. Epub 2010 Aug 5.

40. Katona C, Chiu E, Adelman S, et al. Declaração de consenso da seção de psiquiatria geriátrica da Associação Mundial de Psiquiatria sobre ética e capacidade em pessoas idosas com doença mental; World Psychiatric Association section of old age psychiatry consensus statement on ethics and capacity in older people with mental disorders. Rev Psiq Clín. 2010;37:157-61.

41. Sá LV, Oliveira RA. Autonomia: uma abordagem interdisciplinar. Rev Saude Etica Just. 2007;12(1/2):5-14.

42. Eike-Henner W. Incompetent patient, substitute decision making and quality of life: some ethical considerations. Medscape J Med. 2008;10:237.

43. Almeida EHR. Dignidade, autonomia do paciente e doença mental. Rev Bioetica. 2010;18:381-95.

44. Gessert CE. The problem with autonomy. Minnesota Med. 2008;91(4):40-2. 